Journal of Inclusion Phenomena and Macrocyclic Chemistry

Original Research Article

\title{
A polyrotaxane gel using boronic acid-appended $\gamma$-cyclodextrin as a hybrid cross-linker
}

Wataru Uchida, ${ }^{a}$ Maiki Yoshikawa, ${ }^{\text {a }}$ Tomohiro Seki, ${ }^{a}$ Ryotaro Miki, ${ }^{a}$ Toshinobu Seki, ${ }^{\text {a }}$ Takashi Fujihara, ${ }^{\mathrm{b}}$ Yoshihiro Ishimaru, ${ }^{\mathrm{c}}$ Yuya Egawa ${ }^{* a}$

a. Faculty of Pharmacy and Pharmaceutical Sciences, Josai University, 1-1 Keyakidai, Sakado, Saitama 350-0295, Japan.

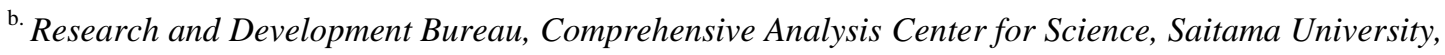
Shimo-ohkubo 255, Sakura-ku, Saitama, Saitama 338-8570, Japan

c. Division of Material Science, Graduate School of Science and Engineering, Saitama University, 255 Shimo-ohkubo, Sakura-ku, Saitama, Saitama, 338-8570, Japan

* yegawa@josai.ac.jp

$\underline{\text { Tel }+81-49-271-7686}$

$\underline{\text { Fax }+81-49-271-7714}$

\begin{abstract}
A boronic acid-appended $\gamma$-cyclodextrin (BA-CyD) was synthesized as a hybrid cross-linker of polyvinyl alcohol (PVA) to form a new type of hydrogel. The CyD moiety of BA-CyD forms an inclusion complex with the PVA chain to produce a mechanically interlocking structure. At the same time, the BA moiety of BA-CyD forms covalent bonds with the 1,3-diol moieties of PVA. On the basis of these two modes of interaction, the hybrid cross-linker connects two PVA chains, thus resulting in the formation of a hydrogel. To investigate the possibility of this hydrogel becoming the basis for an intelligent material for drug delivery, sugar-responsive drug release from the hydrogel was demonstrated.
\end{abstract}

\section{Keywords}

cyclodextrin; boronic acid; polyrotaxane; gel; intelligent material; sugar response.

\section{Introduction}

Until recently, gels have been classified into two types on the basis of the differences in the type of cross-linking within the polymer network.[1] The first type comprises chemically cross-linked gels in which the cross-linking consists of covalent bonds. The second type comprises physically 
cross-linked gels in which the cross-linking is provided by intermolecular interactions such as hydrogen bonding and hydrophobic aggregation.

Nowadays, a third type of cross-linked gels has been developed on the basis of mechanically interlocked structures of polyrotaxane.[2] Rotaxane, which is a type of supramolecular structure, composed of a dumbbell-shaped molecule and a cyclic molecule that covers the bar of the dumbbell.[3, 4] When many cyclic molecules cover a polymer chain, the structure is called polyrotaxane.[5] A prime example of polyrotaxane has been developed by Harada et al.,[6, 7] used $\alpha$-cyclodextrin $(\alpha-\mathrm{CyD})$, which is a cyclic oligo-saccharide comprising six glucose units, and polyethylene glycol (PEG) to form a complex. They also modified the terminus of PEG with a bulky stopper for $\alpha-C y D$ such as the dinitrophenyl group.

By using this type of polyrotaxane, pioneering work for developing the third gel type has been conducted by Ito et al., who used the hydroxyl groups of $\alpha-\mathrm{CyD}$ to form covalent bonds within the polyrotaxane.[8] The chemically cross-linking $\alpha$-CyD can slide onto the PEG chains, thus giving rise to a mechanically interlocked structure that is different from ordinary chemically cross-linked gels. The delocalization of cross-linking point produces a gel with unique features, such as large expansibility and viscoelasticity.[9, 10] Given their structure, gels belonging to the third type have been called slide ring gels.

In this article, we report the synthesis of boronic acid-appended CyD (BA-CyD), which we used as a hybrid cross-linker of polyvinyl alcohol (PVA) chains to prepare an unprecedented gel. We expected that BA-CyD would connect two PVA chains via two driving forces (Fig. 1).

The first driving force leads to the formation of a covalent bond between the BA moiety and repeated 1,3-diol moieties of PVA.[11,12] BA derivatives show affinity for 1,2- or 1,3-diol groups to form five- or six-membered cyclic esters. Many attempts have been made to develop sugar sensors on the basis of the esterification between a boronic acid moiety and diols from sugars.[13, 14]

The second driving force leads to a mechanical interlocking between the PVA chain and CyD rings. In our study, we used $\gamma$-CyD, which is composed of eight glucose units. We made this choice because a $\gamma$-CyD ring is large enough for a PVA chain to thread the cavity of the $\gamma$-CyD structure.[15] In this context, the CyD moiety would be prevented from 'sliding' off the PVA chain because covalent bonding between the BA moiety and PVA chain would act as a stopper of the CyD rings. If the two driving forces, namely, chemical bonding and mechanically interlocking, work simultaneously, BA-CyD will cross-link two PVA chains and induce gelation.

We synthesized BA-CyD by a click chemistry approach consisting of copper(I)-catalyzed azide-alkyne cycloaddition. Thus, synthesized BA-CyD was mixed with PVA in an aqueous medium to obtain a hydrogel. We then investigated the features of this hydrogel. Furthermore, we explored the effect of polyol compounds on the latter process because polyols compete with 
1,3-diols in the reaction with the BA moiety and should induce the disintegration of the gel. Gel polyol response was investigated to test the possibility of achieving sugar-induced drug release with the aim of developing intelligent materials for drug delivery.[16]

\section{Experimental}

Materials

$\gamma$-CyD was purchased from Junsei Chemical Co. Ltd. (Tokyo, Japan). PVA ( 500 polymerization degree, 86.0-90.0 mol\% saponification degree), sodium azide, copper(II) sulfate pentahydrate and uranine (sodium fluorescein) were obtained from Wako Pure Chemical Industries Ltd. (Osaka, Japan). p-Toluenesulfonyl chloride, 2-propyn-1-ol, 1-(3-dimethylaminopropyl)-3-ethylcarbodiimide hydrochloride (EDC) and sodium L-ascorbate, tris[(1-benzyl-1H-1,2,3-triazol-4-yl)methyl]amine (TBTA) were obtained from Tokyo Kasei Kogyo (TCI) Co. Ltd. 4-Carboxyphenylboronic acid and $N$-(2-hydroxyethyl)piperazine- $N$ '-(2-ethanesulfonic acid) (HEPES) were purchased from Sigma-Aldrich Japan (Tokyo, Japan).

\section{Apparatus}

Liquid-state ${ }^{1} \mathrm{H}-\mathrm{NMR}$ measurements were conducted with a Varian 400-MR (Agilent Technologies, California, USA). Solid-state ${ }^{13} \mathrm{C} \mathrm{CP} / \mathrm{MAS}$ NMR was recorded at $125.7 \mathrm{MHz}$ on a Bruker AVANCE500T (Bruker BioSpin K.K, Kanagawa, Japan) with a sample spinning rate of $\sim 8 \mathrm{KHz}$. Chemical shifts were referenced to the carbonyl carbon of glycine used as an external standard. Differential scanning calorimetry (DSC) was conducted using a Thermo Plus2 series (Rigaku Corporation, Tokyo, Japan). A sample was heated in an aluminum pan at a heating rate of $5 \mathrm{~K} / \mathrm{min}$ under nitrogen atmosphere. Turbidity was monitored with a V-530 UV-Vis spectrometer (JASCO Corporation, Tokyo, Japan) by using sample absorbance at $700 \mathrm{~nm}$. The amount of released fluorescein was monitored using Spectra Max M5e multiplate reader (Molecular Devices Japan, Tokyo, Japan).

\section{Synthesis}

Mono-6-O-(p-toluenesulfonyl)- $\gamma$-CyD. $\gamma$-CyD (13.0 g, $10.0 \mathrm{mmol})$ was dried under reduced pressure at $90^{\circ} \mathrm{C}$ for $2 \mathrm{~h}$ and then dissolved in dehydrated pyridine $(400 \mathrm{~mL})$. A solution of $p$-toluenesulfonyl chloride $(5.00 \mathrm{~g}, 26.0 \mathrm{mmol})$ in pyridine $(100 \mathrm{~mL})$ was slowly added to the $\gamma$-CyD solution at $4{ }^{\circ} \mathrm{C}$, and the resulting reaction mixture was stirred for $1 \mathrm{~h}$ at the said 
temperature. To quench the reaction, water $(50.0 \mathrm{~mL})$ was added to the solution. The mixture was concentrated under reduced pressure and was poured into acetone $(1.00 \mathrm{~L})$. As a result of this operation, a precipitate of the crude desired compound formed, and this precipitate was filtered through a glass filter and washed with acetone repeatedly. The crude compound was purified by column chromatography by using a highly porous polystyrene gel (Diaion HP-20, Mitsubishi Chemical, Japan) and a $20 \%$ methanol aqueous solution as eluent. The yield was $30.4 \%$. ${ }^{1} \mathrm{H}-\mathrm{NMR}$ (400 MHz, DMSO-d $d_{6} \delta 7.75$ (d, 2H, phenyl group), $\delta 7.43$ (d, 2H, phenyl group), $\delta 5.79(\mathrm{~m}, 16 \mathrm{H}$, CyD OH-2, OH-3), $\delta 4.87$ (m, 8H, CyD H-1), $\delta 4.53$ (m, 7H, CyD OH-6), $\delta 3.76-3.35$ (m, 32H, CyD H-3, H-5, H-6), $\delta$ 3.35-3.19 (m, 16H, CyD H-2, H-4), $\delta 2.40$ (s, 3H, toluene $\mathrm{CH}_{3}$ ) and MS (FAB, positive mode, matrix: glycerol) $\mathrm{m} / \mathrm{z}: 1451.5$ (expected $\mathrm{m} / \mathrm{z}$ of $[\mathrm{M}+\mathrm{H}]^{+}: 1451.5$ ).

Mono-(6-azide-6-deoxy)- $\gamma$-CyD. Mono-6-O-(p-toluenesulfonyl)- $\gamma$-CyD (4.00 g, $2.76 \mathrm{mmol})$ and sodium azide $(1.96 \mathrm{~g}, 30.2 \mathrm{mmol})$ were dissolved in water $(60.0 \mathrm{~mL})$ at $80^{\circ} \mathrm{C}$, and the resulting solution was stirred for $3 \mathrm{~h}$ at the same temperature. After the reaction solution was allowed to cool to room temperature, it was added drop-wise to acetone $(1.00 \mathrm{~L})$. This operation led to the formation of a precipitate, the crude product, which was filtered and washed with acetone. The crude product was purified by column chromatography using Diaion HP-20 and a 50\% methanol aqueous solution as eluent. Yield was 81.2\%. ${ }^{1} \mathrm{H}-\mathrm{NMR}\left(400 \mathrm{MHz}, \mathrm{DMSO}-d_{6}\right) \delta 5.77(\mathrm{~m}, 16 \mathrm{H}$, CyD OH-2, OH-3), $\delta 4.87$ (m, 8H, CyD H-1), $\delta 4.53$ (m, 7H, CyD OH-6), $\delta 3.74-3.45$ (m, 32H, CyD H-3, H-5, H-6) and $\delta 3.40-3.22$ (m, 16H, CyD H-2, H-4)

Benzoic acid, 4-borono-, 2-propyn-1-yl ester. 4-Carboxyphenylboronic acid (1.66 g, $10.0 \mathrm{mmol})$, 2-propyn-1-ol (5.82 mL, $100 \mathrm{mmol})$, triethylamine $(2.80 \mathrm{~mL}, 20.0 \mathrm{mmol})$ and EDC $(3.83 \mathrm{~g}, 20.0$ $\mathrm{mmol}$ ) were dissolved in DMF (40.0 mL) and stirred at room temperature in $\mathrm{N}_{2}$ atmosphere for 5 h. The mixture was then concentrated under reduced pressure and a saturated aqueous solution of $\mathrm{NaHCO}_{3}(80.0 \mathrm{~mL})$ was added to it. The aqueous phase was extracted with ethyl acetate $(80.0 \mathrm{~mL}$ $\times 3$ ). The organic layers were combined and washed with brine then dried and filtered. The filtrate was subjected to rotary evaporation, and this process led to the isolation of the crude product, which was purified by silica gel chromatography with, as eluent, a mixed solvent with the following components and ratios: chloroform/methanol/acetic acid =100/5/1. Yield was 39.2\%.

${ }^{1} \mathrm{H}-\mathrm{NMR}\left(400 \mathrm{MHz}, \mathrm{DMSO}-d_{6}\right), \delta 8.31$ (s, 2H, BOH), $\delta 7.91$ (m, 4H, phenyl group), $\delta 4.94$ (s, 2H, $\left.\mathrm{OCH}_{2} \mathrm{C} \equiv\right), \delta 3.61(\mathrm{~s}, 1 \mathrm{H}, \mathrm{C} \equiv \mathrm{CH})$ and $\mathrm{MS}(\mathrm{FAB}$, positive mode, matrix: glycerol) $\mathrm{m} / \mathrm{z}: 261$ (expected $\mathrm{m} / z$ of $\left[\mathrm{M}+\mathrm{H}+\mathrm{glycerol}-\mathrm{H}_{2} \mathrm{O}\right]^{+}: 261$ ). The observed peak was attributed to a cyclic ester form with glycerol. 
BA-CyD. Mono-(6-azide-6-deoxy)- $\gamma$-CyD (2.00 g, $1.51 \mathrm{mmol})$, benzoic acid, 4-borono-, 2 propyn-1-yl ester $(0.309 \mathrm{~g}, 1.51 \mathrm{mmol}), \mathrm{CuSO}_{4} \cdot 5 \mathrm{H}_{2} \mathrm{O}(18.9 \mathrm{mg}, 75.6 \mu \mathrm{mol})$, sodium ascorbate (59.9 mg, $0.303 \mathrm{mmol})$ and TBTA $(40.1 \mathrm{mg}, 75.6 \mu \mathrm{mol})$ were dissolved in DMF/ $\mathrm{H}_{2} \mathrm{O}(1 / 1, \mathrm{v} / \mathrm{v})$. The mixture was stirred at room temperature in $\mathrm{N}_{2}$ atmosphere for $6 \mathrm{~h}$. The reaction solution was concentrated under reduced pressure then added to acetone $(1.00 \mathrm{~L})$. As a consequence, a precipitate of the crude desired product formed and was filtered and purified by column chromatography using a selective boron ion exchange resin (Amberlite IRA743 free base, Organo, Japan) and a $10 \%$ acetic acid aqueous solution as eluent. The eluate was first concentrated then purified by column chromatography by using Diaion HP-20 and a $20 \%$ methanol aqueous solution as eluent. The yield was 68.3\%. ${ }^{1} \mathrm{H}-\mathrm{NMR}(400 \mathrm{MHz}, \mathrm{DMSO}-\mathrm{d} 6), \delta 8.28$ (m, 2H, phenyl group), $\delta 8.18(\mathrm{~s}, 1 \mathrm{H}$, triazole), $\delta 7.89(\mathrm{~m}, 2 \mathrm{H}$, phenyl group), $\delta 5.82(\mathrm{~m}, 16 \mathrm{H}, \mathrm{CyD} \mathrm{OH}-2, \mathrm{OH}-3)$, $\delta 4.87$ (m, 8H, CyD H-1), $\delta 4.53$ (m, 7H, CyD OH-6), $\delta 3.76-3.47$ (m, 32H, CyD H-3, H-5, H-6), $\delta$ 3.45-3.11 (m, 16H, CyD H-2, H-4) and MS (FAB, positive mode, matrix: glycerol) $\mathrm{m} / \mathrm{z}: 1582.6$ (expected $m / z$ of $\left[\mathrm{M}+\mathrm{H}+\text { glycerol }-2 \mathrm{H}_{2} \mathrm{O}\right]^{+}: 1582.5$ ). BA-CyD was detected as a cyclic ester composed of BA-CyD and glycerol. Elemental analysis, calculated for $\mathrm{C}_{58} \mathrm{H}_{102} \mathrm{~N}_{3} \mathrm{BO}_{50}$ [BA-CyD.7 $\left.\mathrm{H}_{2} \mathrm{O}\right]:$ C 42.16, H 6.22, N 2.54\%, found: C 42.18, H 5.97, N 2.41\%

\section{Preparation of hydrogels composed of PVA and BA-CyD}

To prepare a hydrogel with a 1/3 (BA-CyD/vinyl alcohol) molar ratio, an aqueous solution of BA-CyD $(0.20 \mathrm{M}, 300 \mu \mathrm{L})$ was mixed with an aqueous solution of PVA $(80 \mathrm{mg} / \mathrm{mL}, 100 \mu \mathrm{L})$, and the resulting reaction mixture was kept at room temperature for $14 \mathrm{~h}$. To prepare a hydrogel with a 1/12 (BA-CyD/vinyl alcohol) molar ratio, the same procedure was implemented using a BA-CyD aqueous solution that had $1 / 4$ the concentration reported above $(0.050 \mathrm{M}, 300 \mu \mathrm{L})$.

\section{Turbidity measurements}

The hydrogel was prepared in the 1/12 (BA-CyD/vinyl alcohol) molar ratio and subsequently lyophilised. The freeze-dried hydrogel $(10 \mathrm{mg})$ was suspended in a buffer solution (20 mM HEPES, pH 7.4, $2.00 \mathrm{~mL}$ ) in a cell for absorption spectrometry, and the turbidity of the prepared sample was measured by recording its absorbance at $700 \mathrm{~nm}$. A stock solution $(200 \mu \mathrm{L}, 1.00 \mathrm{M})$ of polyol (catechol, D-fructose, or D-glucose) was added to the suspension to obtain a final 100 $\mathrm{mM}$ concentration of the polyol. The change in turbidity was followed. 
To prepare a hydrogel with a 1/12 (BA-CyD/vinyl alcohol) molar ratio containing sodium fluorescein, an aqueous solution of BA-CyD $(0.050 \mathrm{M})$ and sodium fluorescein $(10 \mu \mathrm{g} / \mathrm{mL})$ was prepared. Subsequently, $300 \mu \mathrm{L}$ of this solution and an aqueous solution of PVA ( $80 \mathrm{mg} / \mathrm{mL}, 100$ $\mu \mathrm{L}$ ) were mixed together in a glass vial, and the resulting mixture was kept at room temperature for $14 \mathrm{~h}$. To start a release experiment, a buffer solution (100 mM HEPES, pH 7.4, $2.00 \mathrm{~mL}$ ) of $100 \mathrm{mM}$ polyol (D-fructose or D-glucose) was added to the glass vial and was kept at $37^{\circ} \mathrm{C}$. After a predetermined time, the content of the vial was mixed through gentle inversions of the vial. The supernatant $(100 \mu \mathrm{L})$ was then collected, and the amount of fluorescein released was monitored with a microplate reader $\left(\lambda_{\mathrm{ex}}=493 \mathrm{~nm}, \lambda_{\mathrm{em}}=519 \mathrm{~nm}\right)$.

\section{Results and discussion}

Synthesis of BA-CyD and preparation of hydrogels

Mono-6- $O$-( $p$-toluenesulfonyl)- $\gamma$-CyD was converted to mono-(6-azide-6-deoxy)- $\gamma$-CyD, which was then used for a click chemistry reaction consisting of copper(I)-catalyzed azide-alkyne cycloaddition. BA-CyD was successfully prepared by the reaction between mono-(6-azide-6-deoxy)- $\gamma$-CyD and benzoic acid, 4-borono-, 2-propyn-1-yl ester. This type of azide-alkyne cycloaddition reaction will provide us many variations in future studies.

To fabricate a hydrogel, BA-CyD was used as a hybrid cross-linker. Some previous reports described how simply mixing PVA and native CyD in aqueous solution did not produce a PVA-based hydrogel. Harada et al. reported that the simple mixing of PVA with native $\alpha$-CyD, $\beta$-CyD or $\gamma$-CyD does not lead to the formation of an inclusion complex.[17] Hernández et al. have also confirmed that although mixing PVA with native $\gamma-\mathrm{CyD}$ in an aqueous solution generated turbidity, it did not result in the formation of a hydrogel or a crystalline precipitate. [15] To obtain a PVA-based hydrogel, they repeated freeze-thaw cycles of aqueous PVA solutions, and upon adding $\gamma$-CyD to them, they detected the formation of an inclusion complex between PVA and $\gamma$-CyD in the hydrogel. The researchers found that in the PVA-based hydrogel they prepared, the cross-linking interaction between PVA chains consists of hydrogen bonding, thus indicating that this gel is physically cross-linked by definition.

In this study, we attempted to prepare an unprecedented PVA hydrogel wherein BA-CyD acts as a hybrid cross-linker. Initially, we set the molar ratio of $\mathrm{BA}-\mathrm{CyD}$ to vinyl alcohol to be one to three because we estimated that one $\gamma$-CyD molecule covers three repeated units of vinyl alcohol, which is equal to the length of six atoms of the main chain of PVA. This estimation is based on the fact that $\alpha-C y D$, which has the same depth as $\gamma-C y D,[18]$ includes two ethylene glycol units, which is equal to the length of six atoms.[19] When the said molar ratio was $1 / 3$, a semitransparent gel formed, which displayed fluidity (Fig. 2, left). When the BA-CyD/vinyl 
alcohol molar ratio was reduced to $1 / 12$, a white-colored, cloudy gel formed that did not display fluidity (Fig. 2, right). The white color was due to Mie scattering, which indicates that the cross-linked aggregated clusters are of similar size or larger than the wavelengths of visible light.

Notably, a smaller amount of BA-CyD produced a harder gel, which is an apparently counterintuitive find because a larger proportion of cross-linker usually leads to the formation of harder gels in chemically cross-linked systems. We propose that this result is evidence of the two driving forces of gel formation working simultaneously. When the BA-CyD/vinyl alcohol molar ratio is high, many segments of the PVA molecule are covered by CyD moieties; consequently, the BA moiety cannot bind the 1,3-diol unit of PVA (Fig. 3a). If not so, when many BA moieties are appended on PVA, the PVA chain cannot thread the cavity of CyD because of the blockage by the BA groups 'branching' out of the PVA chain (Fig. 3b). Although we assume that the first interaction inhibits the second, it is difficult to judge which mechanism is the main factor determining the formation of a soft gel when the BA-CyD/vinyl alcohol ratio is 1/3.

Inclusion of PVA by the CyD moiety of $B A-C y D$

To obtain information on the structure of the hydrogel, a hydrogel was prepared using a 1/12 (BA-CyD/vinyl alcohol) molar ratio and subsequently lyophilized. The hydrogel in the vial was frozen at $-20^{\circ} \mathrm{C}$, and it was lyophilized with a freeze dryer (Labconco BTD, Asahi Life Science Co., Ltd. Saitama, Japan ) at room temperature for 1 day. The lyophilized gel was utilized for a DSC experiment. The thermogram obtained for the gel was compared with those obtained for PVA, BA-CyD and a physical mixture of PVA and BA-CyD. The thermogram of PVA (Fig. 4a) shows a broad signal because of an endothermic process at around $195^{\circ} \mathrm{C}$, which corresponds to the melting point of PVA. Melting is mainly the result of the collapse of the crystal structure because of the cleavage of the hydrogen bonds between PVA chains. In the same experimental conditions, this endothermic signal was not observed in the lyophilized gel composed of PVA and BA-CyD (Fig. 4d), thus indicating that the partially crystalline structure of PVA-based on hydrogen bonding was not present in the lyophilized gel. These results suggest that the driving force of the gelation was not the hydrogen bonding between PVA chains.

We then conducted solid-state ${ }^{13} \mathrm{C} \mathrm{CP/MAS} \mathrm{NMR} \mathrm{spectrometry} \mathrm{experiments} \mathrm{on} \mathrm{the}$ lyophilized gel to try and obtain evidence for inclusion of PVA by the CyD moiety. In the ${ }^{13} \mathrm{C}$ CP/MAS NMR spectrum of PVA (Fig. 5a), the signals at 60-80 ppm are due to the CH carbon of the PVA chain. They are split into three peaks because of the difference in the triad tacticity.[20] The signal at around $40 \mathrm{ppm}$ is assigned to the $\mathrm{CH}_{2}$ carbon of the PVA chain. Furthermore, two peaks can be observed at around 20 and $170 \mathrm{ppm}$; these peaks are due to the residual acetyl groups.[20] In general, PVA is synthesized by the polymerization of vinyl acetate, and the 
resulting polyvinyl acetate is saponificated to obtain PVA; however, some acetyl groups are retained to maintain the water solubility of the polymer.[21] In the maker's property information of the PVA used in this study, the saponification degree is $86.0-90.0 \mathrm{~mol} \%$. The PVA used in this study showed an absorption peak at around $1720 \mathrm{~cm}^{-1}$ in the infrared spectrum, thus confirming the existence of the acetyl group (Fig. S1). The signals at around 20 and $170 \mathrm{ppm}$ are assigned to the $\mathrm{CH}_{3}$ carbon and the $\mathrm{C}=\mathrm{O}$ carbon of the acetyl group, respectively.[20]

In the spectrum of the lyophilized gel, both peaks at around 20 and $170 \mathrm{ppm}$ appeared broadened and shifted to a lower magnetic field (Fig. 5c). We consider these changes to be caused by the fact that acetyl groups are included by the CyD moiety even though they are not involved in ester formation. The ester formation between vinyl alcohol and the BA moiety may induce some changes in the spectrum; however, the acetyl groups appear not to be involved in ester formation with the BA moiety. The postulation of the inclusion of the acetate residue by the CyD moiety is reasonable because an inclusion complex of $\gamma-\mathrm{CyD}$ and polyvinyl acetate has been reported previously.[22]

No other clear-cut evidence of the formation of an inclusion complex between PVA and BA-CyD could be seen from the NMR spectrometry data. For example, the peak at around 40 ppm due to the $\mathrm{CH}_{2}$-carbon of PVA (Fig. 5a) shows a change in chemical shift in the spectrum of the lyophilized gel (Fig. 5c). This change may be caused by inclusion by the CyD moiety or by ester formation with the BA moiety; however, it is not clear which of the two scenarios is the one responsible for the observed peak shift.

Furthermore, some peaks derived from BA-CyD did not show any change in the spectrum of the lyophilized gel (Fig. 5c). We tried obtaining evidence of the inclusion of PVA by the CyD moiety by comparing the shape of NMR signals. In fact, it is known that these shapes provide some information on the included guest molecule. The ${ }^{13} \mathrm{C} C P / M A S$ NMR spectrum of unoccupied native $\gamma-\mathrm{CyD}$ is characterized by split peaks due to an asymmetry in the structure for the ring (Fig. S2), and the inclusion of a guest reduces such asymmetry.

As a result, peaks display a smooth appearance.[15] The NMR spectrum of the lyophilized gel (Fig. 5c) shows smooth signals, thus suggesting that the CyD moiety formed some kind of inclusion complex. However, the spectrum of BA-CyD (Fig. 5b) also displays smooth peaks, thus possibly indicating the inclusion of the $\mathrm{BA}$ moiety by the $\mathrm{CyD}$ moiety. Consequently, the smoothness of the peak in Fig. 5(c) cannot be a decisive piece of evidence of the inclusion of a PVA chain by BA-CyD.

By summarizing the analysis detailed above, we obtained two possible pieces of evidence that indicate the inclusion of PVA by BA-CyD: the disappearance of the PVA melting point in the DSC analysis of the lyophilized gel and the changes in the peaks due to the acetate residue of PVA in the NMR spectrum of the lyophilized gel. 
Binding between PVA and the BA moiety of BA-CyD

The gelation of PVA prompted by the interaction with BA-CyD depends on two driving forces that work simultaneously: inclusion by the CyD moiety and binding by the BA moiety. As mentioned above, simply mixing PVA and native $\gamma$-CyD does not lead to gel formation. Similarly, phenylboronic acid alone does not work as a gelling agent. Mixing PVA and phenylboronic acid in an aqueous solution resulted in the formation of a precipitate but not of a hydrogel (data not shown).

To verify whether the BA moiety plays a role in gel formation, we observed the dissolution behavior of the lyophilized gel in the presence and absence of polyols (Fig. 6). We used catechol, D-fructose and D-glucose as polyols because they show affinity for BA derivatives. To evaluate gel dissolution behavior, we measured the turbidity monitored through absorbance at $700 \mathrm{~nm}$. The lyophilized gel was stirred in buffer solution with and without polyols.

In the absence of polyols, the turbidity remained constant for $15 \mathrm{~min}$, thus indicating that the lyophilized gel powder dissolves slowly in these conditions. In the presence of polyols, the dissolution rate increased. The observed change in rate of dissolution was consistent with the order of the binding constants for the formation of BA derivatives. For example, the binding constants of phenylboronic acid with catechol, D-fructose and D-glucose are 830, 210 and 4.6 $\mathrm{M}^{-1}$, respectively, at $\mathrm{pH}$ 7.5.[23]

The increase in the rate of dissolution associated with the presence of polyols indicates that reversible binding by the BA moiety plays a crucial role in the cross-linking process that leads to gel formation.

Polyol-responsive fluorescein release from the gel

Polyols promoted the dissolution of the lyophilized gel, thus offering the possibility of devising intelligent materials for chemical sensing and drug delivery.[24] For example, a sugar-responsive drug release system would be useful for the insulin treatment of patients with diabetes. [25]

We prepared a gel in the presence of fluorescein that act as a drug molecule model, and recorded the release profile of fluorescein from the gel. When the gel was immersed in a buffer solution, fluorescein was released gradually. In these conditions, $20 \%$ of fluorescein was determined to have been released after $8 \mathrm{~h}$ while the gel kept its initial shape (Fig. 7, left). In the presence of D-fructose, the gel was completely disintegrated, and $100 \%$ of fluorescein was released after $3 \mathrm{~h}$ (Fig. 7, center). By contrast, the addition of D-glucose did not appear to enhance the erosion of the gel (Fig. 7, right.) 
From the standpoint of developing a drug delivery system for patients with diabetes, the response of the gel to D-glucose should be increased. For this purpose, introducing other BA derivatives will be useful. For example, phenylboronic acid with an electron-withdrawing group shows higher affinity for D-glucose than BA.[16, 26] Furthermore, the fact that a click reaction between an azide and an alkyne was used to introduce the BA moiety in $\gamma$-CyD means that a wide range of BA derivatives are available to modify CyD.

\section{Conclusions}

We successfully obtained a PVA gel by using BA-CyD as a hybrid cross-linker and confirmed that chemical bonding by the BA moiety and mechanical interlocking by the CyD moiety work together to drive the formation of the gel. To the best of our knowledge, this study is the first report on the concept of a hybrid cross-linker that creates two types of cross-linking modes at the same time.

In ordinary gels, a chemical cross-linker generates the same chemical bonds at both ends. Even if two kinds of chemical bonds are created by a bifunctional cross-linker, the gels that this cross-linker produces are still categorised as chemically cross-linked. In the case of physically cross-linked gels, two polymer chains share the same interaction, such as hydrogen bonding and hydrophobic interaction.

On the contrary, the two ends of BA-CyD are implicated in different types of cross-linking interactions: a mechanically interlocked structure involving the $\mathrm{CyD}$ group and covalent chemical bonds involving the BA moiety; the resulting gel meets the definition of the third type of cross-linked gels. This novel concept of hybrid cross-linker seems to hold considerable promise for developing new types of gels.

\section{References}

1. Hennink, W.E., van Nostrum, C.F.: Novel crosslinking methods to design hydrogels. Adv. Drug Deliv. Rev. 64, 223-236 (2012).

2. Ito, K.: Novel cross-Linking concept of polymer network: Synthesis, structure, and properties of slide-Ring gels with freely movable junctions. Polym. J. 39, 489-499 (2007).

3. Araki, J., Ito, K.: Recent advances in the preparation of cyclodextrin-based polyrotaxanes and their applications to soft materials. Soft Matter. 3, 1456-1473 (2007).

4. Girek, T.: Cyclodextrin-based rotaxanes. J. Incl. Phenom. Macrocycl. Chem. 74, 1-21 (2012).

5. Takata, T.: Polyrotaxane and polyrotaxane network: Supramolecular architectures based on the concept of dynamic covalent bond chemistry. Polym. J. 38, 1-20 (2006). 
6. Harada, A., Li, J., Kamachi, M.: The molecular necklace: a rotaxane containing many threaded a-cyclodextrins. Nature. 356, 325-327 (1992).

7. Harada, A.: Cyclodextrin-based molecular machines. Acc. Chem. Res. 34, 456-464 (2001).

8. Okumura, Y., Ito, K.: The polyrotaxane gel: A topological gel by figure-of-eight cross-links. Adv. Mater. 13, 485-487 (2001).

9. Kato, K., Yasuda, T., Ito, K.: Viscoelastic properties of slide-ring gels reflecting sliding dynamics of partial chains and entropy of ring components. Macromolecules. 46, 310-316 (2013).

10. Ito, K.: Slide-ring materials using cyclodextrin. Chem. Pharm. Bull. 65, 326-329 (2017).

11. Watahiki, R., Sato, K., Suwa, K., Niina, S., Egawa, Y., Seki, T., Anzai, J.: Multilayer films composed of phenylboronic acid-modified dendrimers sensitive to glucose under physiological conditions. J. Mater. Chem. B. 2, 5809-5817 (2014).

12. Anzai, J.: Recent progress in electrochemical biosensors based on phenylboronic acid and derivatives. Mater. Sci. Eng. C. 67, 737-746 (2016).

13. Egawa, Y., Seki, T., Takahashi, S., Anzai, J.I.: Electrochemical and optical sugar sensors based on phenylboronic acid and its derivatives. Mater. Sci. Eng. C. 31, 1257-1264 (2011).

14. James, T.D.: Self and directed assembly: people and molecules. Beilstein J. Org. Chem. 12, 391405 (2016).

15. Hernández, R., Rusa, M., Rusa, C.C., López, D., Mijangos, C., Tonelli, A.E.: Controlling PVA Hydrogels with $\gamma$-Cyclodextrin. Macromolecules. 37, 9620-9625 (2004).

16. Seki, T., Abe, K., Egawa, Y., Miki, R., Juni, K., Seki, T.: A pseudopolyrotaxane for glucose-responsive insulin release: The effect of binding ability and spatial arrangement of phenylboronic acid group. Mol. Pharm. 13, 3807-3815 (2016).

17. Harada, A., Hashidzume, A., Takashima, Y.: Cyclodextrin-based supramolecular polymers. Chem. Soc. Rev. 38, 875-882 (2009).

18. Harada, A., Hashidzume, A., Yamaguchi, H., Takashima, Y.: Polymeric rotaxanes. Chem. Rev. 109, 5974-6023 (2009).

19. Harada, A., Li, J., Kamachi, M., Kitagawa, Y., Katsube, Y.: Structures of polyrotaxane models. Carbohydr. Res. 305, 127-129 (1997).

20. Terao, T., Maeda, S., Saika, A.: High-Resolution Solid-state ${ }^{13}$ C NMR of poly(viny1 alcohol): enhancement of tacticity splitting by intramolecular hydrogen bonds. Macromolecules. 16, 15351538 (1983).

21. Dai, L., Yu, S.: Effect of degree of saponification on structural and property change of poly (vinyl alcohol) fibers. Polymer. Adv. Tech. 14, 449-457 (2003).

22. Uyar, T., Aslan, E., Tonelli, A.E., Hacaloglu, J.: Pyrolysis mass spectrometry analysis of poly(vinyl acetate), poly(methyl methacrylate) and their blend coalesced from inclusion compounds formed with $\gamma$-cyclodextrin. Polym. Degrad. Stab. 91, 1-11 (2006). 
23. Yan, J., Springsteen, G., Deeter, S., Wang, B.: The relationship among $\mathrm{p} K_{\mathrm{a}}$, $\mathrm{pH}$, and binding constants in the interactions between boronic acids and diols - It is not as simple as it appears. Tetrahedron. 60, 11205-11209 (2004).

24. Culver, H.R., Clegg, J.R., Peppas, N.A.: Analyte-responsive hydrogels: Intelligent materials for biosensing and drug delivery. Acc. Chem. Res. 50, 170-178 (2017).

25. Meng, F.H., Zhong, Z.Y., Feijen, J.: Stimuli-responsive polymersomes for programmed drug delivery. Biomacromolecules. 10, 197-209 (2009).

26. Matsumoto, A., Ishii, T., Nishida, J., Matsumoto, H., Kataoka, K., Miyahara, Y.: A synthetic approach toward a self-regulated insulin delivery system. Angew. Chem. Int. Ed. 51, 2124-2128 (2012).

\section{Figures}
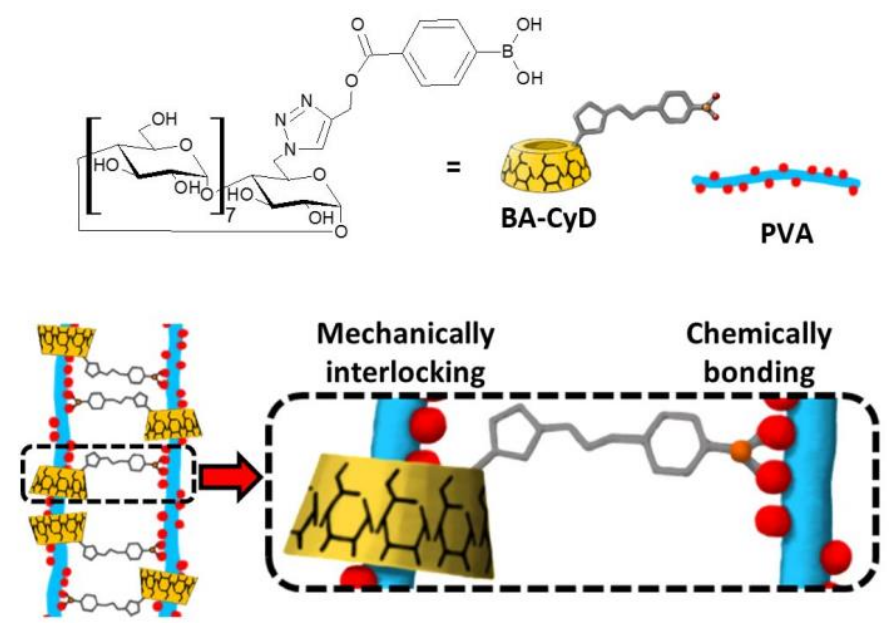

Fig. 1 Chemical structure of BA-CyD and schematic illustration of a PVA-based gel cross-linked by BA-CyD.

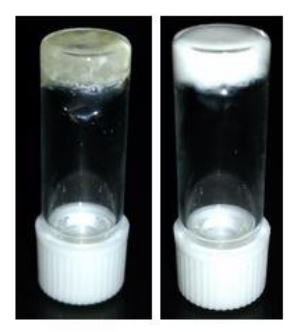

Fig. 2 Hydrogels composed of PVA and BA-CyD. A gel prepared with a 1/3 molar ratio between BA-CyD and vinyl alcohol (left). A gel prepared with a 1/12 molar ratio between BA-CyD and vinyl alcohol (right). The hydrogels were prepared in vials, and the vials were turned upside down. 


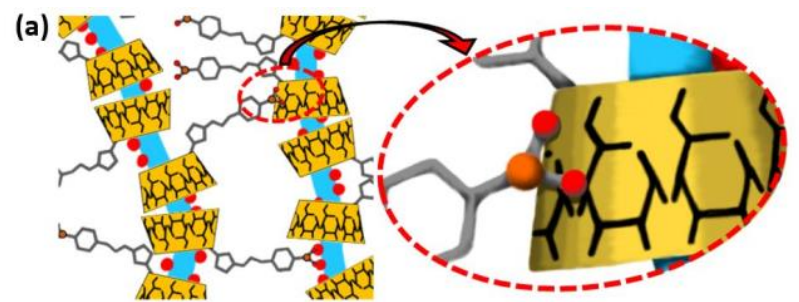

(b)

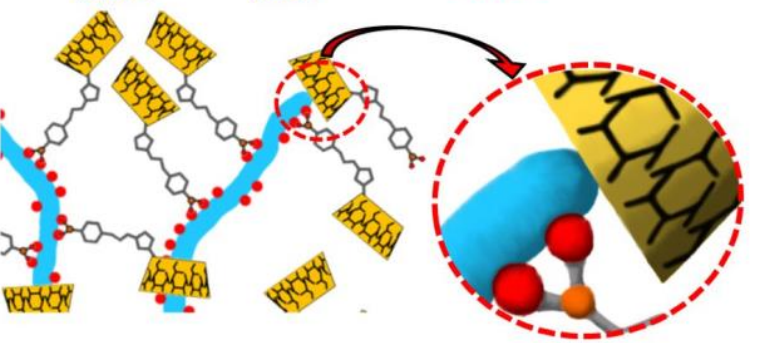

Fig. 3 Proposed mechanisms that would explain why a high BA-CyD/vinyl alcohol molar ratio produces a soft gel. (a) The CyD moiety inhibits bond formation between the BA moiety and PVA. (b) The BA moiety protruding from the PVA chain inhibits the CyD moiety from sliding onto PVA to form an inclusion complex.

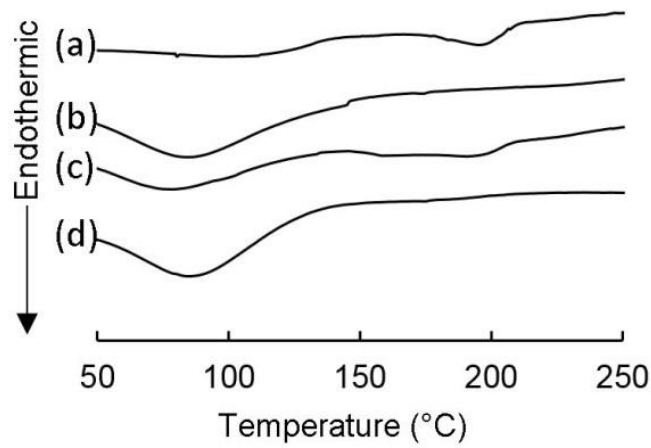

Fig. 4 DSC thermograms of PVA (a), BA-CyD (b), a physical mixture of PVA and BA-CyD (c) and a lyophilized gel prepared using a 1/12 (BA-CyD/vinyl alcohol) molar ratio (d). 


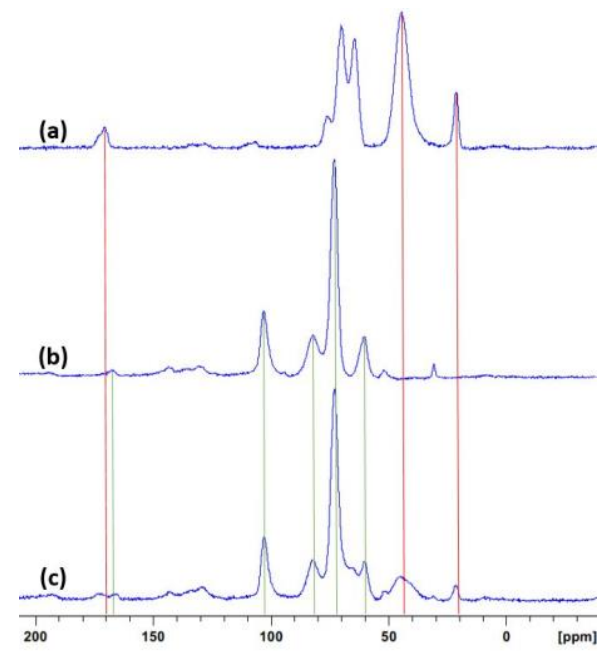

Fig. $5{ }^{13} \mathrm{C}$ CP/MAS NMR spectrum of PVA(a), BA-CyD (b) and a lyophilized gel prepared using a 1/12 (BA-CyD/vinyl alcohol) molar ratio (c).

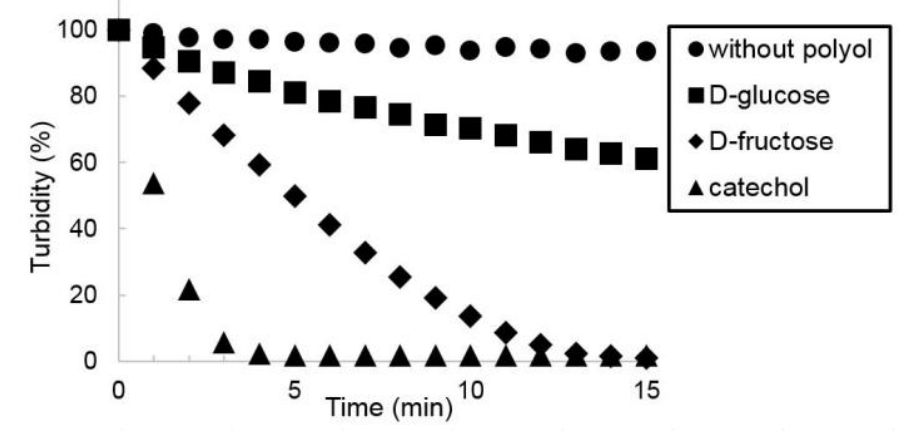

Fig. 6 Turbidity changes of the suspension of the lyophilized gel prepared with a molar ratio of 1/12 (BA-CyD/vinyl alcohol) in the presence and absence of polyols $\left(\mathrm{pH} 7.4,37^{\circ} \mathrm{C}, \mathrm{n}=1\right)$. 

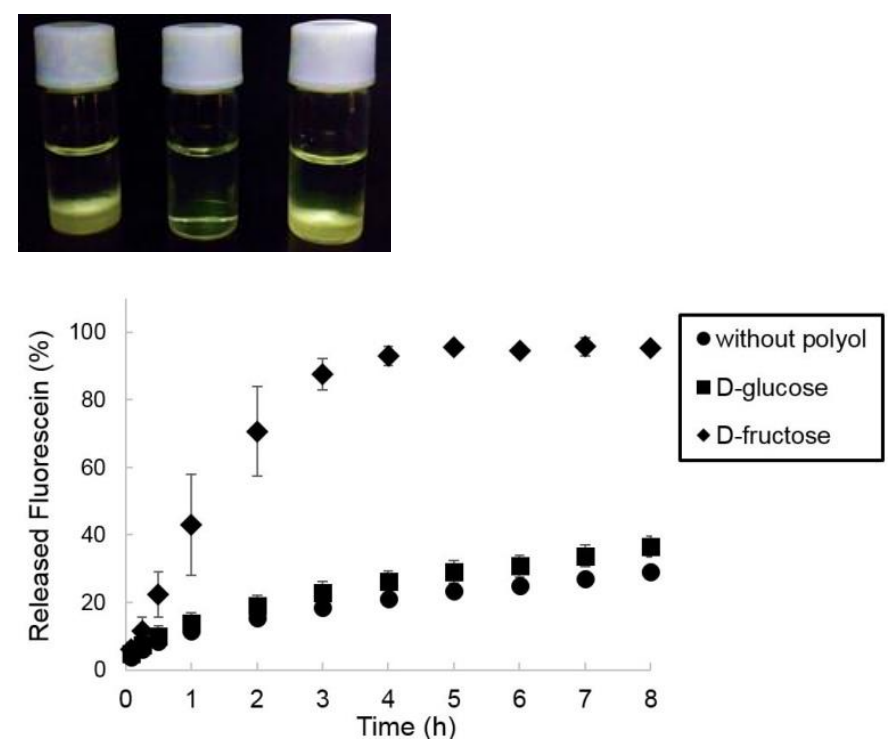

Fig. 7 Release profiles of fluorescein from a hydrogel prepared with a 1/12 (BA-CyD/vinyl alcohol) molar ratio (above), and the effect of polyols $\left(\mathrm{pH} 7.4,37^{\circ} \mathrm{C}, \mathrm{n}=3\right.$ ). Picture of the vial without polyol (left), with D-fructose (center) and D-glucose (right) after the release experiments (below). 


\section{Supporting Information}

\section{A polyrotaxane gel using boronic acid-appended $\mathrm{Y}^{-}$cyclodextrin as a hybrid cross-linker}

Wataru Uchida, ${ }^{a}$ Maiki Yoshikawa, ${ }^{a}$ Tomohiro Seki, ${ }^{a}$ Ryosuke Miki, ${ }^{a}$ Toshinobu Seki, ${ }^{a}$

Takashi Fujihara, b Yoshihiro Ishimaru, c Yuya Egawa*a

a. Faculty of Pharmacy and Pharmaceutical Sciences, Josai University, 1-1 Keyakidai, Sakado, Saitama 350-0295, Japan.

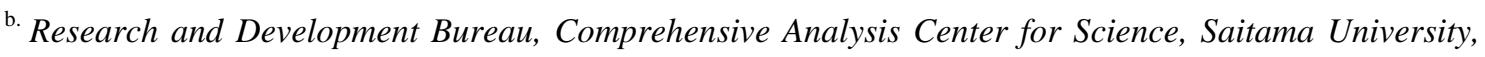
Shimo-ohkubo 255, Sakura-ku, Saitama, Saitama 338-8570, Japan

c. Division of Material Science, Graduate School of Science and Engineering, Saitama University, 255 Shimo-ohkubo, Sakura-ku, Saitama, Saitama, 338-8570, Japan

\section{Contents}

IR spectra

Solid-state ${ }^{13} \mathrm{C}$ CP/MAS NMR spectrum of $\gamma$-CyD

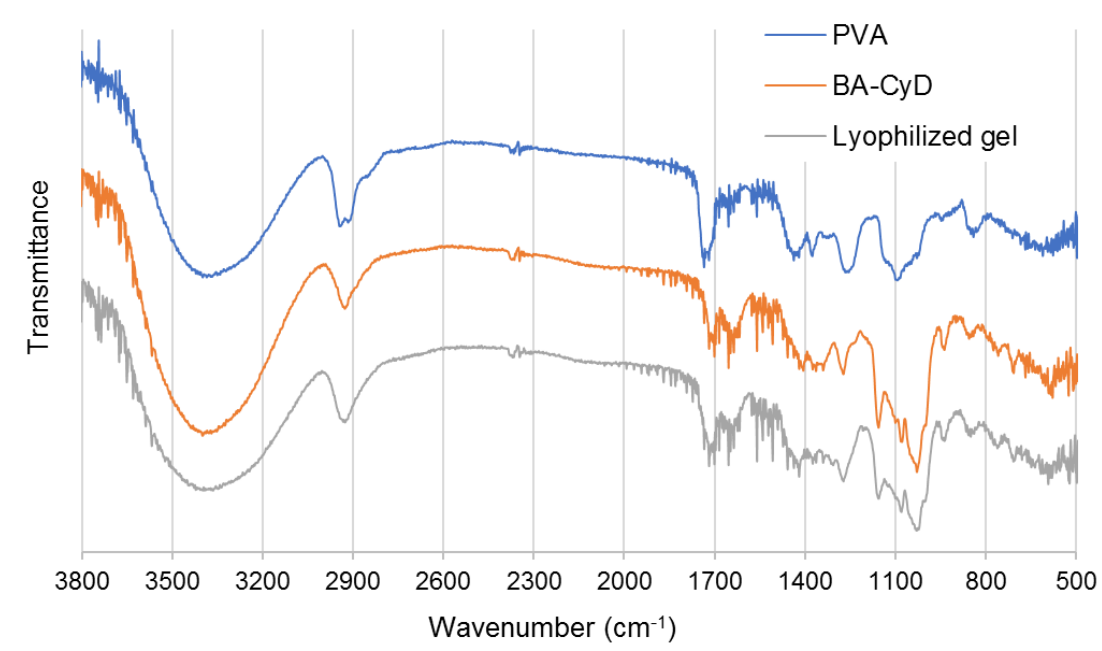

Fig. S1. IR spectra of PVA, BA-CyD and the lyophilised gel prepared using a 1/12 (BA-CyD/vinyl alcohol) molar ratio. 


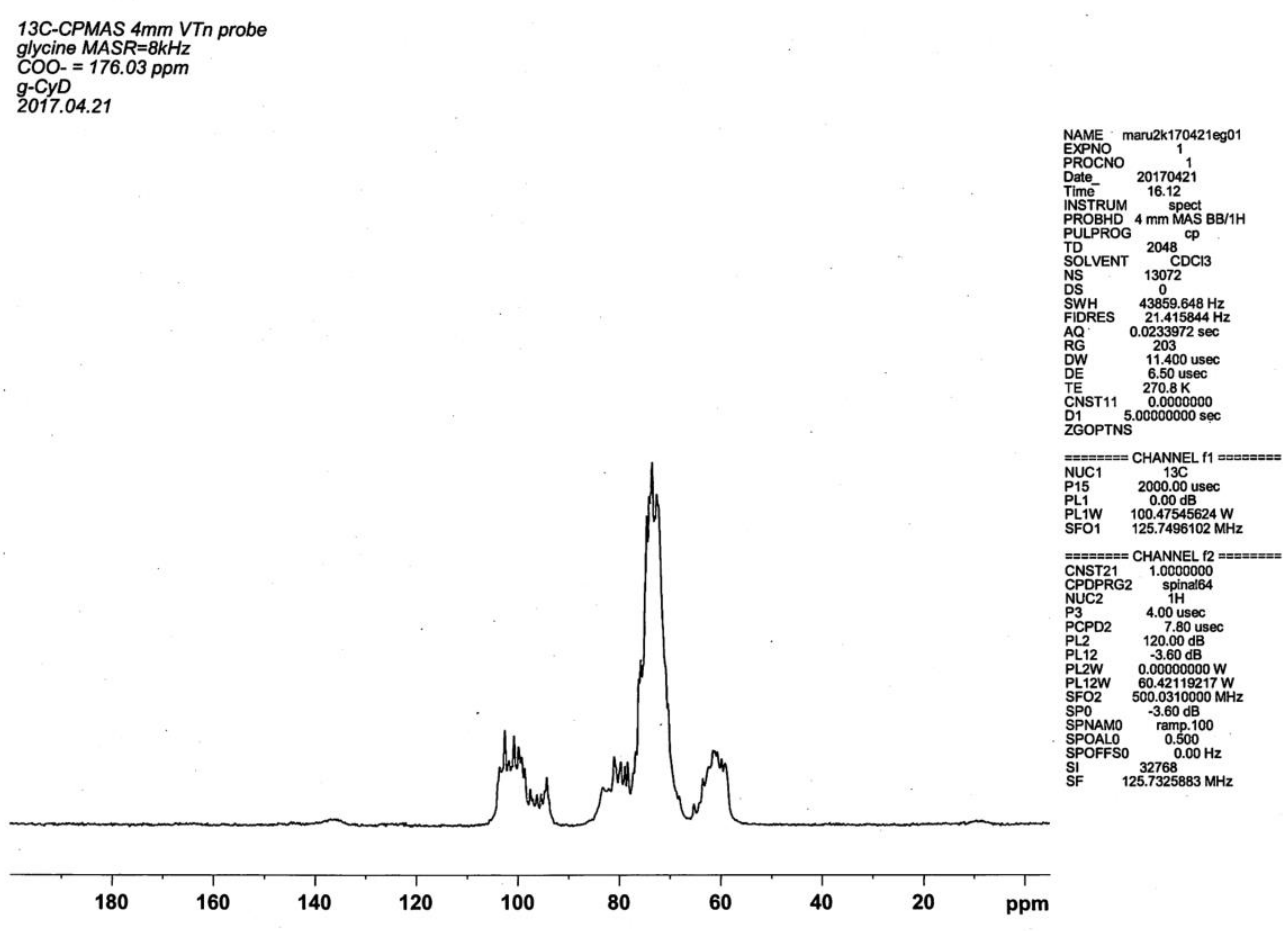

Fig. S2. Solid-state ${ }^{13} \mathrm{C} C \mathrm{CP} / \mathrm{MAS}$ NMR spectrum of native $\gamma$-CyD. 